\title{
The post-hematopoietic cell transplantation microbiome: relationships with transplant outcome and potential therapeutic targets
}

\author{
Yannouck F. van Lier,, ${ }^{1,2}$ Marcel R.M. van den Brink, ${ }^{3,4}$ Mette D. Hazenberg ${ }^{1,2,5}$ \\ and Kate A. Markey ${ }^{3,4}$
}

${ }^{1}$ Department of Hematology, Amsterdam UMC, Amsterdam, the Netherlands; ${ }^{2}$ Department of Experimental Immunology, Amsterdam Institute for Infection and Immunity (All), Cancer Center Amsterdam, Amsterdam UMC, Amsterdam, the Netherlands; ${ }^{3}$ Adult Bone Marrow Transplantation Service, Department of Medicine, Memorial Sloan Kettering Cancer Center, New York, NY, USA; ${ }^{4}$ Department of Medicine, Weill Cornell Medical College, New York, NY, USA and ${ }^{5}$ Department of Hematopoiesis, Sanquin Research, Amsterdam, the Netherlands

Haematologica 2021

Volume 106(8):2042-2053

\section{Correspondence:}

KATE MARKEY

markeyk@mskcc.org

Received: January 11, 2021.

Accepted: April 1, 2021.

Pre-published: April 22, 2021.

https://doi.org/10.3324/haematol.2020.270835

(C)2021 Ferrata Storti Foundation

Material published in Haematologica is covered by copyright. All rights are reserved to the Ferrata Storti Foundation. Use of published material is allowed under the following terms and conditions:

https://creativecommons.org//icenses/by-nc/4.0/legalcode. Copies of published material are allowed for personal or internal use. Sharing published material for non-commercial purposes is subject to the following conditions:

https://creativecommons.org/licenses/by-nc/4.0/legalcode, sect. 3. Reproducing and sharing published material for commercial purposes is not allowed without permission in writing from the publisher.

\section{ABSTRACT}

M icrobiota injury occurs in many patients undergoing allogeneic hematopoietic cell transplantation, likely as a consequence of conditioning regimens involving chemo- and radiotherapy, the widespread use of both prophylactic and therapeutic antibiotics, and profound dietary changes during the peri-transplant period. Peri-transplant dysbiosis is characterized by a decrease in bacterial diversity, loss of commensal bacteria and single-taxon domination (e.g., with Enterococcal strains). Clinically, deviation of the post-transplant microbiota from a normal, high-diversity, healthy state has been associated with increased risk of bacteremia, development of graft-versus-host disease and decreases in overall survival. A number of recent clinical trials have attempted to target the microbiota in allogeneic hematopoietic cell transplantation patients via dietary interventions, selection of therapeutic antibiotics, administration of pre- or pro-biotics, or by performing fecal microbiota transplantation. These strategies have yielded promising results but the mechanisms by which these interventions influence transplant-related complications remain largely unknown. In this review we summarize the current approaches to targeting the microbiota, discuss potential underlying mechanisms and highlight the key outstanding areas that require further investigation in order to advance microbiota-targeting therapies.

\section{Introduction}

Allogeneic hematopoietic cell transplantation (HCT) is the oldest form of cellular therapy for patients with hematologic malignancies, and utilizes stem cell-containing grafts from carefully selected donors to invoke immunological anti-malignancy effects. This procedure remains high risk for patients, as transplantationrelated complications, such as infection and graft-versus-host disease (GvHD), are causes of high morbidity and mortality among allogeneic HCT recipients. ${ }^{1,2}$ The incidence of acute GvHD in human leukocyte antigen-matched donors remains $30-35 \%{ }^{3}$ The role of the microbiome in transplant-related complications has been a point of investigation in the field for decades, but advances in sequencing technology and our capacity to study the function of intestinal microbial communities have accelerated this interest in recent years. Approaches to conserve or recover a healthy microbiome in transplant patients are increasingly being used in experimental settings with the goal of improving overall transplant outcome.

Pre-transplant conditioning regimens expose the gastrointestinal tract to high doses of chemo- and radiotherapy, resulting in loss of integrity of the intestinal epithelium and inflammatory damage which often results in mucositis. ${ }^{4}$ Clinical manifestations of mucositis include mouth sores, pain during eating or swallowing, nausea, intestinal cramping, bloating and diarrhea, which commonly minimize a patient's oral dietary intake. Mucositis is also thought to increase the risk of micro- 
bial translocation from the lumen of the oral cavity or gastrointestinal tract into the systemic circulation, which has led to the widespread use of antibiotic prophylaxis in allogeneic HCT recipients in whom prolonged mucositis and co-incident neutropenia are common. Patients also have high rates of exposure to broad-spectrum empiric antibiotics, which are initiated in response to fever in the neutropenic period. Microbial sources of these neutropenic febrile episodes are rarely proven but the consequences of untreated severe infection in this group of patients are such that it is standard practice to initiate broad-spectrum empiric therapy when a fever occurs. ${ }^{5}$

Acute GvHD is classically described as a three-step process. ${ }^{6,7}$ The first step involves host tissue injury, resulting in release of damage-associated patterns (DAMPS; uric acid, ATP, heparin sulfate, HMGB-1, interleukin [IL]33) and pathogen-associated patterns (PAMPS; lipopolysaccharides [LPS]), which further stimulate the production of inflammatory cytokines such as tumor necrosis factor [TNF]- $\alpha$, IL- 1 and IL- 6 . The second step comprises priming and expansion of alloreactive donor lymphocytes, predominantly $\mathrm{T}$ cells, which are recruited to the host tissues; hematopoietic and non-hematopoietic antigen-presenting cells interact with alloreactive lymphocytes, skewing $\mathrm{CD}^{+} \mathrm{T}$ lymphocytes towards a $\mathrm{T}$ helper (Th1) or Th17 phenotype that produce inflammatory cytokines such as interferon (IFN)- $\gamma$ and IL-17, activating cytotoxic $\mathrm{CD}^{+} \mathrm{T}$ cells, and stimulating their proliferation. The third step involves recruitment and activation of additional (innate) effector cells, such as macrophages and neutrophils, which amplify the cytokine production ('cytokine storm') and orchestrate further apoptotic tissue damage.

Systemic, high-dose corticosteroids are the first-line treatment for acute GvHD and are successful in about two-thirds of the cases. ${ }^{8}$ Patients with severe or steroidrefractory GvHD have a dismal prognosis, with longterm survival rates reported between $5-30 \% .^{9}$ This has led to persistent efforts to identify new therapies for GvHD.

Our current knowledge of the composition of the intestinal microbial community, in both health and disease, is largely based on the analysis of fecal samples using an amplicon-based sequencing approach that targets the variable regions of the prokaryotic $16 \mathrm{~S}$ ribosomal RNA gene. $16 \mathrm{~S}$ rRNA sequencing allows us to measure microbial composition (i.e., the presence of certain taxa) and compute the summary measure $\alpha$-diversity (commonly measured by the Simpson or Shannon index), which has been used in a large number of transplant studies that have associated microbiota abnormalities with transplant outcome..$^{10}$ A second method is metagenomic sequencing, which involves untargeted ('shotgun') metagenomic sequencing of all the genes present in a given sample. Multiple technologies are available for metagenomic sequencing, but they can be broadly grouped into either short-read (e.g., Illumina) or long-read (e.g., PacBio, Nanopore) platforms. ${ }^{11}$ Independently of the sequencing method, metagenomic sequencing provides reliable species-level taxonomy, as well as profiling of the functional capacity of the intestinal microbial community as a whole, by identifying genes that confer microbial function (e.g., fatty acid synthesis or degradation). Metagenomic sequencing can thus be a powerful hypothesis-generating tool and can enhance our understanding of potential mechanisms underpinning associations between particular microbial compositions and clinical outcome.

\section{The healthy microbiome and intestinal homeostasis}

The human microbiome constitutes a diverse collection of bacteria, viruses, archaea and eukaryotic microbes that inhabit all parts of the body but predominantly reside in the gut. ${ }^{12}$ Bacteria represent the largest group within the intestinal microbiome and most species belong to the Firmicutes or Bacteroidetes phyla with smaller contributions from the Proteobacteria, Actinobacteria, Fusobacteria and Verrucomicrobia phyla. The dynamic ecosystem within the intestinal tract is continuously subjected to environmental changes induced by diet, medication and disease of the host, which can dramatically alter microbial composition. ${ }^{13-15}$ Factors important in maintaining homeostasis and preventing pathogen outgrowth and translocation include: an intact gut epithelium that physically separates the luminal microorganisms from the underlying host tissue; an extra layer of protection, provided by the mucus layer that is produced by goblet cells, to hinder bacterial translocation and prevent mucosal barrier injury; the anaerobic commensals that predominate in the gut microbiota community of healthy individuals and that provide resistance against colonization by pathogens. The products of anaerobic commensals further prevent pathogen outgrowth; molecules derived from commensal bacteria, which can signal to Paneth cells via toll-like receptors (TLR) 2, 4, 5 and 9, and induce production of antimicrobial peptides. ${ }^{16}$ Antimicrobial peptides, such as defensins and regenerating gene (REG) III proteins, shape the microbial composition by sequestering essential nutrients and permeabilizing bacterial membranes; the commensals, which in addition to mediating resistance to colonization by potential pathogens, also produce metabolites that are essential micronutrients for the human host.

Microbial metabolites include vitamins $\left(\mathrm{K}, \mathrm{B}_{12}\right)$, shortchain fatty acids (SCFA) such as butyrate, propionate and acetate, which are the products of indigestible dietary fiber fermentation, aryl hydrocarbon receptor ligands called indoles generated through tryptophan metabolism and secondary bile acids. ${ }^{17-20}$ These microbial metabolites are locally important for maintenance of epithelial integrity and stimulation of the mucosal immune system, but can also exert effects on peripheral organs via secretion into the systemic circulation. ${ }^{21-24}$ SCFA act via several pathways to maintain homeostasis: they serve as an energy source for colonocytes, promote mucus secretion, enhance expression of tight junction proteins to improve epithelial integrity, induce anti-inflammatory cytokines such as IL-10 and IL-18, and promote differentiation of anti-inflammatory regulatory $T$ cells via engagement with G-protein coupled receptors (GPR) 43, 41 and 109a on both epithelial cells and immune cells. ${ }^{25}$ Indoles also have immune-modulating potential. For example, they can induce IL-22 production by different aryl hydrocarbon receptor-expressing lymphocytes, such as Th17 cells, natural killer T cells, $\gamma \delta \mathrm{T}$ cells and innate lymphoid cells, which in turn enhances epithelial barrier function and promotes antimicrobial peptide production. ${ }^{26}$ 


\section{Relationship of the microbiome with transplant-related toxicities}

There are many contributors to the disruption of the intestinal microbiota observed in the peri-transplant period, including conditioning, diet changes, and exposure to antibiotics (Figure 1). Early after transplantation, there is a loss of microbial diversity as well as a shift towards a microbiome dominated by Enterococci, Streptococci or, in fewer cases, Proteobacteria, at the expense of anaerobic populations, such as Bacteroides, Clostridium and Bifidobacterium. Intestinal domination, here defined as a relative abundance of $\geq 30 \%$ of a single taxonomic unit, is associated with exposure to certain antibiotics and increases the risk of blood stream infection by the respective dominating taxon. ${ }^{27,28}$

Decreases in fecal $\alpha$-diversity can be observed even prior to HCT in some patients, but deepens in the days following transplantation, and is associated with the timing and type of antibiotic treatment..$^{27,29 \cdot 34}$ Indeed, clinical studies have shown a reduction of bacterial burden and loss of fecal $\alpha$-diversity following cancer treatment, e.g., for acute myeloid leukemia, which is consistent with the dysbiosis observed pre-transplant. $27,29,0,355-39$ Loss of fecal bacterial diversity is recognized in transplant patients across different geographic locations as was recently documented in a large, observational study that included 8,767 stool samples from 1,362 patients, collected at four different centers in three different continents..$^{30}$ This study also reinforced a previously recognized association between intestinal diversity during the peri-engraftment period (days 7-21 post-HCT) and transplant outcome: low microbial diversity is associated with significantly higher transplantation-related mortality, GvHD-related mortality and lower overall survival. ${ }^{40,41}$ The prevalence of intestinal domination was high in this cohort: it was detected in more than $75 \%$ of the samples collected 1 week after transplantation, with Enterococcus domination being most prevalent. In an earlier study, Enterococcus domination was associated with lower overall survival and higher GvHD-related mortality. ${ }^{42}$ Indeed, perturbations of the microbiome are particularly overt in patients with acute GvHD. ${ }^{37,42-47}$ Pre-clinical studies in mice have demonstrated that intestinal microbiota play an important role in the development of acute GvHD and, vice versa, acute GvHD itself can aggravate intestinal dysbiosis. A study by Varelias and colleagues found that the dysbiotic microbiome of IL-17-deficient mice induced hyperacute GvHD and that this effect was transferable to wildtype mice during co-housing experiments. ${ }^{48} \mathrm{~A}$ second study showed that increases in major histocompatibility complex class II (MHCII) expression on intestinal epithelial cells was microbiota-dependent, and that this upregulation process, which was essential for the initiation of lethal acute GvHD, could be abrogated by antibioticmediated bacterial depletion. ${ }^{49}$ After initiation of acute GvHD, GvHD-mediated tissue damage includes the destruction of Paneth cells, which actively shape the microbial community via production of $\alpha$-defensins. ${ }^{50}$ Loss of Paneth cells decreased the expression of $\alpha$ defensins and propagated expansion of pathogenic bacteria (Escherichia coli), which accelerated GvHD. An abundance of Enterococcus species has also been associated with acute GvHD severity: mice with an Enterococcusdominated microbiome experienced worse GvHD and had an inferior survival. ${ }^{42}$ In contrast, the presence of intestinal Blautia, a genus from the butyrate-producing Lachnospiraceae family, appeared to have protective capacities against acute GvHD development both in mice and humans. ${ }^{41}$

Changes in gut microbiota composition have not only been associated with the development and severity of acute GvHD, but have also been linked to other transplant-related complications. For example, analysis of the fecal microbiota composition early (day 0 to 21 ) after allogeneic HCT identified a cluster of bacteria, dominated by Eubacterium limosum, which was associated with a decreased risk of relapse or progressive disease. ${ }^{51}$

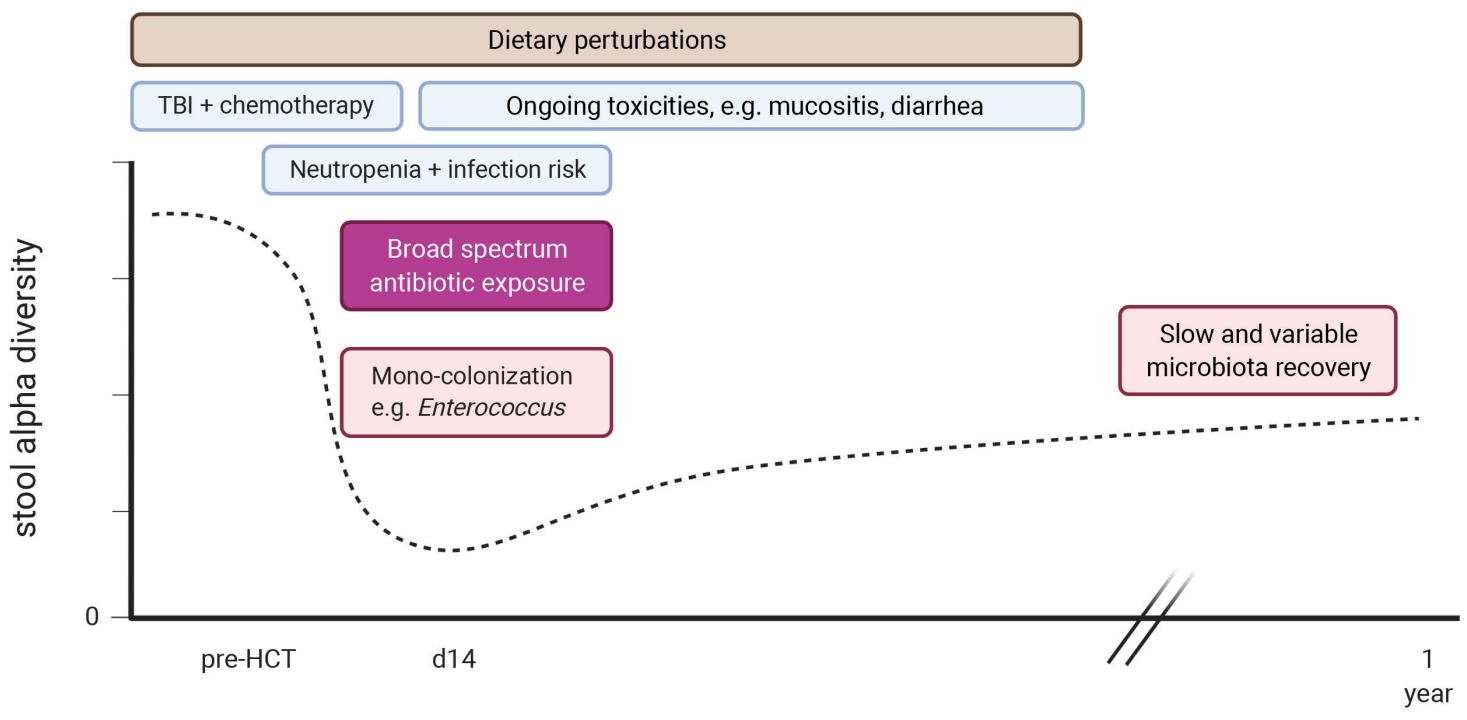

Time relative to $\mathrm{HCT}$

Figure 1. Timeline of potential microbiota perturbations after allogeneic hematopoietic cell transplantation. HCT: hematopoietic cell transplantation; TBI: total body irradiation. Created with BioRender.com 
Additionally, features of the intestinal microbiome have been associated with the development of pulmonary infiltrates post-transplant..$^{52} \mathrm{~A}$ further study found that the presence of butyrate-producing bacteria in fecal samples was associated with a lower rate of viral lower respiratory tract infections. ${ }^{53}$

Finally, there is also evidence emerging that early postHCT microbiome damage may remain for some time, and have consequences for the late complications of transplantation. A recent study by our group showed that dysbiosis can still be observed at 100 days after transplantation and that patients who go on to develop chronic GvHD have lower concentrations of circulating SCFA. ${ }^{54}$ As this was a small study of chronic GvHD patients, further work is needed to explore this association.

The studies described above found several links between dysbiotic gut microbiota and transplant-related complications but it remains largely unresolved whether these associations reflect causal relationships. If this is indeed the case, then manipulation of the microbiota - particularly in a fashion that ensures maintenance of anaerobic bacterial taxa and prevents colonization with potential pathogens could be an effective way to reduce acute transplant-related toxicities and also improve the long-term outcomes for allogeneic HCT recipients. At present, potential strategies for microbiota modification include dietary modification, prebiotics, probiotics or postbiotics and fecal microbiota transplantation (FMT) (Table 1).

\section{Potential mechanisms of microbiome-targeting therapies}

Therapies targeting the microbiota could contribute to preserving or restoring intestinal homeostasis by providing resistance to colonization, enhancing mucosal healing, or dampening the inflammatory immune response.

\section{Colonization resistance}

As mentioned above, outgrowth of a single taxon, particularly Enterococcus, is a common feature of the postHCT microbiome and is associated with a poor transplant outcome. In addition to Enterococcus colonization, intestinal expansion of other pathogenic bacteria, such as the Enterobacterales species Klebsiella pneumoniae and Escherichia coli, has been observed in patients who subsequently developed bloodstream infections caused by the respective species. ${ }^{55}$ Domination may negatively affect intestinal homeostasis: (i) indirectly via loss of beneficial anaerobic bacteria, resulting in a drastic reduction of 'health-promoting' metabolites such as SCFA or, (ii) directly, if the dominating species itself compromises the gut epithelium or invokes an inflammatory response from the mucosal immune system.

Enterococcus faecalis (for example) has the capacity to impair epithelial barrier integrity via matrix metalloproteases and can also induce a strong dose-dependent activation of dendritic cells, resulting in enhanced production of the cytokines IL-6, IL-10, IL-12 and TNF- $\alpha .{ }^{56,57}$ A recent mouse study showed that intake of the disaccharide lactose was necessary for Enterococcus expansion whereas a lactose-free diet attenuated the emergence of Enterococcus and reduced the severity of acute GvHD. ${ }^{42}$ These pre-clinical findings need further exploration in clinical trials, for example, of lactose-free diets in the peri-transplant period, or enzymatic supplementation (e.g., with the lactase enzyme (Lactaid), which is cheap and easily available as it is used commonly by individuals with lactose intolerance).

Reintroduction of specific strains via defined bacterial consortia in oral capsule form (commonly known as probiotics) or transfer of a complete (healthy) microbiome via FMT could prevent domination by a single taxon by promoting resistance to colonization. For example, probiotic therapy with Lactobacillus johnsonii prevented Enterococcus domination after bone marrow transplantation in mice, which coincided with attenuation of acute GvHD. ${ }^{47}$ Similarly, FMT effectively eliminated vancomycin-resistant Enterococcus from densely colonized mice, which was mediated by anaerobic bacteria. ${ }^{58}$ More specifically, recolonization with Barnesiella correlated with eradication of vancomycin-resistant Enterococcus. In these studies, successful engraftment of donor strains was a key component of successful treatment. Factors that could influence this process include pre-treatment strategy (i.e., antibiotic decontamination or bowel lavage, with the latter appearing to be more effective), taxonomic configuration of both donor and recipient and possibly also genetic host factors. ${ }^{59 \cdot 61}$

Alternative approaches that bypass the bacterial community itself but may still enhance colonization resistance include supplementation of antimicrobial peptides or administration of specific TLR agonists. For example, TLR7 might be a candidate as activation of TLR7 on dendritic cells induced IL-22 and mediated colonization resistance against vancomycin-resistant Enterococcus. ${ }^{62}$ Whether targeting this pathway could also be beneficial for transplant outcome needs further investigation.

\section{Healing of the mucosal barrier}

The mucosal barrier becomes compromised during the allogeneic HCT process due to the toxic effects of chemoand radiotherapy and subsequent mucositis, as a result of tissue destruction during acute GvHD, opportunistic

Table 1. Strategies for targeting the microbiome.

\begin{tabular}{lll} 
Method & Strategy & Goal \\
Prebiotics (i.e. non-digestible food ingredients) & Supplement essential bacterial nutrients & Preserve protective taxa \\
Optimizing dietary intake & $\begin{array}{l}\text { Stimulate enteral feeding, avoid consumption } \\
\text { of certain nutrients }\end{array}$ & Preserve protective taxa \\
\hline $\begin{array}{l}\text { Antibiotic selection } \\
\begin{array}{l}\text { Probiotics/defined consortia (i.e. selected } \\
\text { viable microorganisms) or FMT }\end{array}\end{array}$ & $\begin{array}{l}\text { Reduce exposure to anaerobe-targeting antibiotics } \\
\text { Actively reintroduce (specific) microbial populations }\end{array}$ & Limit microbial damage \\
\hline Postbiotics (bioactive metabolic compounds) & Supplement products of bacterial fermentation & Replenish beneficial microbial metabolites \\
\hline
\end{tabular}

FMT: fecal microbiota transplantation. 
infections (e.g., cytomegalovirus) or because of the indirect influence of diminished nutritional intake. Dietary carbohydrates normally serve as the main source of nutrition for the gut microbiota, but in their absence the mucus layer can become an alternative source of energy. As an example, Akkermansia muciniphila is thought to have mucolytic capacities and its relative abundance increases in mice with acute GvHD after imipinem/cilastatin treatment. ${ }^{32,63}$ Another study showed that a diet lacking fiber resulted in thinning of the mucus layer, which increased proximity of microbes to the epithelium. ${ }^{64}$ Enteral feeding (diet or prebiotics) could thus enhance intestinal homeostasis by directly stimulating preservation of commensal populations as well as indirectly by preventing damage to the mucus layer. An alternative way to promote the integrity of the mucus layer is by activating goblet cells which are responsible for the formation of the intestinal mucus layer but are often lost upon development of acute GvHD. It was recently described that stimulation of goblet cells via IL-25 could prevent bacterial translocation and dampened inflammation in an experimental acute GvHD model. ${ }^{65}$ Exogenous supplementation of butyrate or indoles was also able to enhance epithelial integrity and mitigate acute GvHD in experimental mouse models whereas deletion of the SCFA receptor GPR43 increased acute GvHD. ${ }^{66-68}$ However, butyrate can have opposite effects and may delay mucosal wound repair by inhibiting proliferation of intestinal epithelial stem/progenitor cells in a dose-dependent manner. ${ }^{69}$ Whether butyrate has a net-protective or net-pathogenic effect may depend on the time after transplantation, the state of underlying tissue damage, or the activation status of effector $\mathrm{T}$ cells and further work is needed to assess the translatability of these preclinical findings to clinical practice and whether they may be targeted for therapeutic benefit.

Another candidate for promoting mucosal repair is IL22. IL-22, secreted by recipient innate lymphoid cells type 3 , protected intestinal stem cells from immune-mediated damage during murine acute GvHD. ${ }^{70}$ Similarly, recombinant IL-22 enhanced intestinal stem cell recovery, stimulated epithelial regeneration and reduced intestinal acute GvHD pathology. However, pro-inflammatory and destructive effects of donor-derived Il-22 have also been described, suggesting a dual, context-dependent role for this cytokine, which needs further investigation. ${ }^{71}$

\section{Immune modulation}

The cycle of dysbiosis, tissue damage and lymphocyte activation that characterizes the early post-transplant environment and is even more exaggerated when acute GvHD has developed, contains many potential pathways for intervention (Figure 2). Therapies that alter the microbiome and thereby break these feed-forward loops may offer an attractive target in GvHD prevention or treatment.

A number of preclinical studies have determined the immunomodulatory properties of microbiome-targeting or microbiome-adjunct therapies. A prebiotic mixture of glutamine, fiber and oligosaccharides (GFO) as well as FMT lowered colonic expression of IL-1 $\beta$ in dextran sodium sulfate-induced colitis in mice. ${ }^{72,73}$ In addition, FMT reduced the MHCII-dependent antigen-presenting capacities of colonic dendritic cells, monocytes and macrophages while simultaneously enhancing IL-10 production by these antigen-presenting cells, as well as in
$\mathrm{CD}^{+} \mathrm{T}$ cells and invariant natural killer $\mathrm{T}$ cells. ${ }^{73} \mathrm{FMT}$ also appeared to limit $\mathrm{CD}^{+} \mathrm{T}$-cell proliferation while increasing the frequency of $\mathrm{FoxP}^{+}$regulatory $\mathrm{T}$ cells. Induction of regulatory $T$ cells was also accomplished by oral administration of a rationally selected mixture of bacterial strains which mitigated experimental colitis and allergic diarrhea and mainly comprised clusters IV, XIVa and XVIII Clostridia. ${ }^{74}$

While it remains to be clarified whether these mechanisms are also involved in FMT-mediated resolution of steroid-refractory acute GvHD, microbial metabolites might be involved in this process. In addition to SCFA, secondary bile acids, such as lithocholic acid and $3 \beta$ hydroxydeoxycholic acid, are other products of microbiota-mediated biotransformation that are thought to have immunomodulatory properties. For example, lithocholic acid, 3ß-hydroxydeoxycholic acid and other bile acid metabolites have been implicated in the induction of regulatory T-cell populations. ${ }^{75,76}$ A number of studies have shown that bile acid metabolism is altered in patients with acute or chronic GvHD, which might impact disease severity. ${ }^{77-79}$ A randomized controlled trial examining the use of the secondary bile acid ursodeoxycholic acid (also known as ursodiol) for prevention of hepatic complications (e.g., veno-occlusive disease) found a lower incidence of severe acute GvHD and intestinal GvHD in the treatment group that used ursodiol from the start of conditioning through 90 days post-transplantation compared with control-treated patients. ${ }^{80}$ Furthermore, a study of patients with recurrent Clostridioides difficile infections $(n=16)$, which included seven allogeneic HCT recipients, successfully used ursodiol to prevent recurrence of disease. ${ }^{81}$ While the effects of ursodiol on the microbiome of allogeneic HCT recipients requires further investigation, these data suggest that therapeutic interventions targeting microbial bile acid metabolism might be worth exploring.

\section{Opportunities for intervention}

Therapies aimed at modulating intestinal microbiota function can target bacterial energy sources (prebiotics, diet), the bacterial community itself (antibiotic choice, probiotics, FMT) or its metabolites (postbiotics). Their protective or therapeutic potential has been studied in a number of interventional, predominantly single-arm studies.

\section{Prevention of damage by antimicrobials: making the best antimicrobial choices}

Antibiotic stewardship is most commonly considered in terms of its benefits in preventing the emergence of pathogens, but broadly speaking it involves the critical evaluation of antibiotic prescribing and use, to protect patients from all harms (including microbiome disruption) caused by the use of antibiotics, while at the same time effectively treating infections. For example, in a retrospective study, Weber and colleagues observed that rifaximin, a broad-spectrum antibiotic with minimal systemic absorption, was equally effective at preventing infectious complications in allogeneic HCT recipients as ciprofloxacin/metronidazole, while sparing bacterial gut communities. ${ }^{82}$ Another retrospective analysis found that treatment of neutropenic fever with broad-spectrum antibiotics, specifically imipenem/cilastatin and 
A Steady state environment

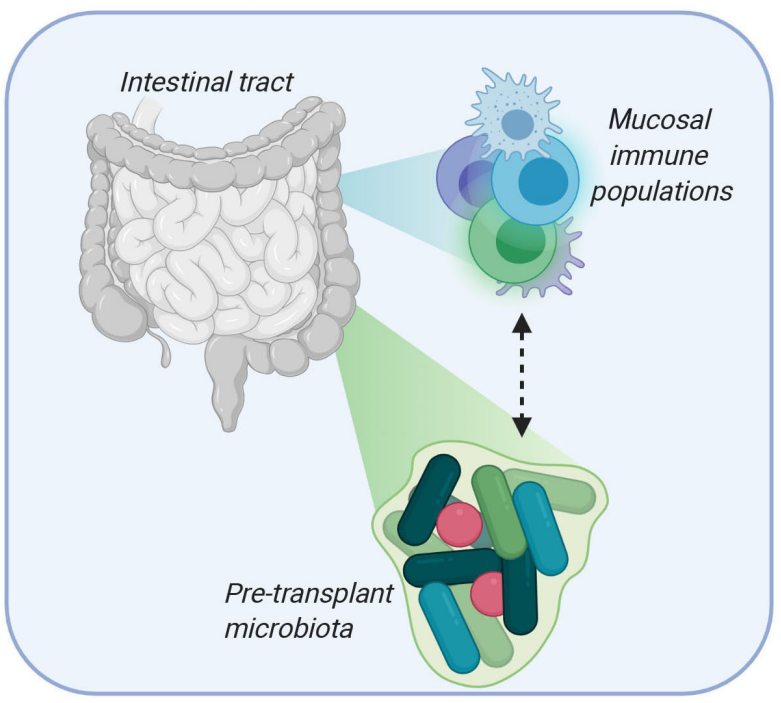

Figure 2. Interactions between the gut microbiota and mucosal immune system in steady state and peri-transplant environments. (A) Pre-transplant interaction between the intestinal tract, mucosal immune system and the microbiome. (B) Peri-transplant interaction between the inflamed intestinal tract, infiltrating donor populations, some of which alloreactive $T$ cells that cause graft-versus-host disease, and the perturbed microbiome, often colonized with potential pathogens, such as Enterococcus faecalis/faecium. APC: antigen-presenting cell; DAMPS: damage-associated patterns; GvHD: graft-versus-host disease; LPS: lipopolysaccharides; PAMPS: pathogen-associated patterns. Created with BioRender.com
B

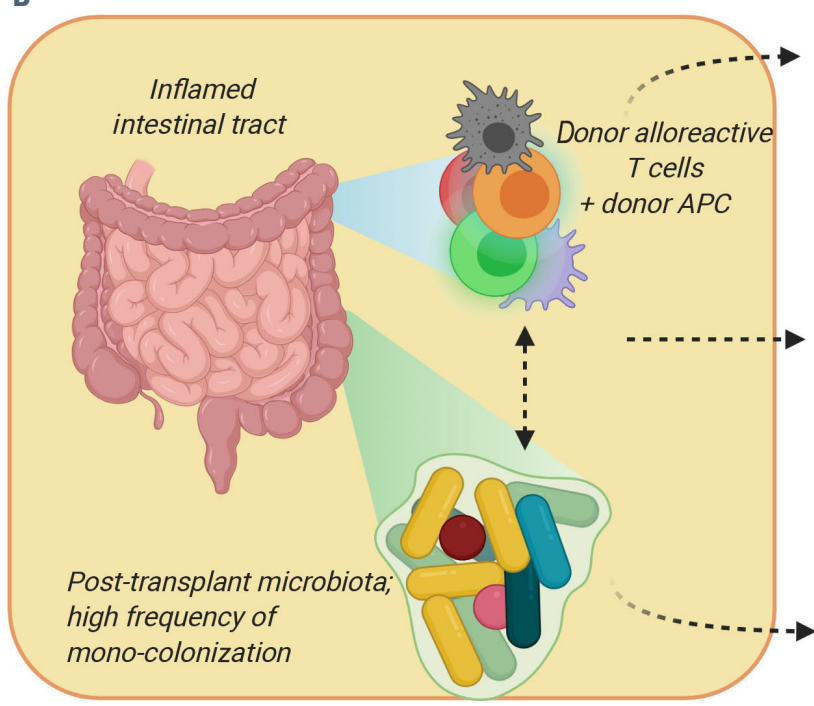

piperacillin/tazobactam, was associated with increased GvHD-related mortality while this was not observed when anaerobic-sparing antibiotics, aztreonam and cefepime, were used. ${ }^{32}$ Of note, shortening the duration of administration of empiric broad-spectrum antibiotics in patients with febrile neutropenia without an identifiable infectious cause has been studied and appears to be safe; however, it remains unclear whether the shortened exposure also reduced intestinal dysbiosis in these patients. ${ }^{83}$ Other strategies that are currently being developed to prevent antibiotic-mediated microbial disruption include molecules that degrade or neutralize antibiotic residues in the intestinal tract. ${ }^{84,85}$

\section{Pre-transplantation optimization of the intestinal microbiome}

Since a relatively preserved microbiome prior to allogeneic HCT translates into a higher chance of a favorable outcome, ${ }^{30}$ it is worth considering a potential role for pre-
Alloreactivity and GvHD

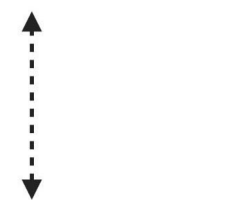

Tissue-damage-associated cytokines; Conditioning related and donor derived

\section{Microbiota-derived DAMPS/PAMPS} e.g. LPS emptive microbiota-targeting therapies. Two studies examined the effect of prebiotic supplementation prior to HCT: a prospective study by Yoshifuji and colleagues demonstrated that adequate intake of resistant starch and GFO from day -7 before allogeneic HCT to day +28 after the transplant reduced the incidence of acute GvHD and preserved populations of butyrate-producing bacteria, while fecal butyrate concentration in samples after transplantation did not differ significantly between GFO-treated patients and historical controls. ${ }^{86}$ The patients with initial high microbial diversity in this cohort appeared more likely to benefit from prebiotic supplementation ( $n=14$ of 30 in the whole cohort). Remarkably, the incidence of late-onset acute GvHD was higher in the prebiotic group, suggesting that there is only a temporary benefit from prebiotic supplementation and that the benefit subsides after cessation of the treatment. Similarly, a second retrospective study observed that patients who received GFO during the same period had higher survival rates early 
after HCT (<day 100) than patients who were not on prebiotic treatment, but this advantage had disappeared 1 year after allogeneic HCT. ${ }^{87}$

A number of small studies have evaluated the safety and feasibility of probiotic use prior to allogeneic HCT but studies evaluating their potency with respect to minimization of transplantation-related complications have not been performed. ${ }^{88,89}$ Oral administration of Lactobacillus rhamnosus GG in mice, starting from day 7 prior to transplantation and continuing throughout the transplantation period, limited bacterial translocation, mitigated acute GvHD and improved survival. ${ }^{90}$ Microbiome analysis was not included in this study, thus further studies are needed to understand the precise mechanism of the observed effects.

Donor FMT effectively reversed antibiotic- and chemotherapy-induced dysbiosis in a murine model, returning the composition of the microbiome to the naive, pre-treatment configuration within 16 days after FMT. ${ }^{11}$ In five allogeneic HCT recipients, donor FMT was successfully used for decolonization of antibiotic-resistant bacteria prior to HCT but the enteric microbiome of these patients was not analyzed..$^{92,93}$

Apart from these studies, data on the impact of microbiota-targeting therapies on the pre-transplant microbiome are scarce. Large, prospective trials are needed to assess to what extent these pre-emptive treatments can overcome treatment-induced microbiota injury and protect against post-transplant complications.

\section{Diet}

It is well-established that dietary changes influence the enteric microbiome and thus it is likely that specific alterations in nutritional support could provide an extra stimulus for the growth and activity of anaerobic bacteria in the peri-transplant setting. ${ }^{94} \mathrm{~A}$ recent study in mice showed that administration of a tyrosine-enriched diet prior to HCT positively modulated the gut microbiome and metabolome, which was accompanied by reduced acute GvHD pathology and improved survival. ${ }^{95}$ Whether a specific diet could help to preserve a healthy microbiome in allogeneic HCT recipients or improve intestinal health prior to allogeneic HCT remains to be elucidated. A study in pediatric patients $(n=20)$ found that patients receiving enteral nutrition after allogeneic HCT had increased fecal diversity, higher concentrations of fecal SCFA and a higher relative abundance of certain butyrateproducing populations than patients receiving parenteral nutrition. ${ }^{96}$ In adult patients $(\mathrm{n}=23)$, fecal microbial diversity was similar in groups receiving either enteral or parenteral nutrition. However, minimal oral intake for a prolonged period was associated with less diversity, as well as less abundance of intestinal Blautia. ${ }^{97}$ Earlier studies had reported the superiority of enteral over parenteral nutrition in terms of transplant-related complications and survival. 98,99

There remains a gap in our knowledge regarding the impact of diet on the microbiome of allogeneic HCT recipients. It has been described that there is a high interpersonal variability in microbiome response to types of nutrition and it might prove difficult to formulate universal dietary recommendations. ${ }^{94,100,101}$ To what extent a specific diet, e.g., devoid of lactose-containing products, could benefit the health of transplant patients needs to be investigated in randomized trials.

\section{Post-transplant recovery of the microbiome}

It is likely that even with the best preventive strategies, some abnormalities in the microbiome will remain after the acute post-transplant period. For those patients with extensive microbial damage, probiotics or (donor) FMT might offer a way to directly repair the microbial community itself. Commercially available probiotic formulations contain bacterial strains predominantly belonging to the Lactobacillus and Bifidobacterium genera. The efficacy of probiotics as a preventive or therapeutic measure has been studied in the context of various disease entities with mixed results. ${ }^{102}$ In fact, the use of probiotics after antibiotic therapy delayed recovery of the indigenous microbiota in healthy individuals. ${ }^{103}$ As for allogeneic HCT recipients, a number of case reports raised safety concerns after systemic infections developed in immunocompromised patients upon probiotic use, even though the bacterial strains that are commonly incorporated in commercially available probiotics are infrequently the cause of bloodstream infections. ${ }^{104-109}$ Probiotic therapy, comprising Lactobacillus rhamnosus GG capsules administered from the time of neutrophil engraftment, did not appreciably alter the microbiome or reduce acute GvHD incidence in a randomized trial $(n=31)$ and was therefore prematurely terminated. ${ }^{110}$

A second option for the re-introduction of a whole community of microorganisms is via transfer of a healthy "previous self" or a donor-derived fecal suspension. In a randomized, controlled trial by Taur et al., allogeneic HCT recipients received either an autologous FMT $(n=14)$, using the patient's banked stool sample that was collected prior to transplant conditioning and carried a high microbial diversity but no intestinal pathogens, or no intervention $(n=11){ }^{29}$ Autologous FMT improved microbial diversity and reinstated commensal members of the patient's gut microbiome which had disappeared after allogeneic HCT. While effective, autologous FMT can be logistically challenging, as it requires upfront collection of a patient's healthy stool; for this reason, most current studies have used related or unrelated healthy donors as the source for FMT.

High success rates have been reported for single or repeated donor FMT in patients to restore symbiosis, eradicate antibiotic-resistant bacteria or treat recurrent Clostridioides difficile infections, which coincidently also relieved GvHD symptoms in one patient. ${ }^{92,93,111-118}$ Resolution of steroid-refractory acute GvHD using donor FMT has been described in a number of case reports. ${ }^{119-122}$ Additionally, a pilot study from our group showed that donor FMT alleviated steroid-refractory or steroid-dependent acute GvHD in ten of 15 participants, which was accompanied by an increase of fecal microbial diversity, expansion of butyrate-producing bacteria, and enhanced donor species engraftment. ${ }^{123}$ The intrinsic and extrinsic factors that facilitate donor species engraftment remain to be elucidated and blinded, randomized clinical trials are needed to determine the additive value of donor FMT in GvHD treatment.

Safety has been one of the major concerns regarding the use of donor FMT in immunocompromised patients. For this reason, FMT trials have, thus far, largely postponed administration until allogeneic HCT recipients have achieved neutrophil engraftment. The number of reported infection-related adverse events associated with donor FMT administration is low but transmission of 
pathogenic microorganisms has been described and can be fatal. ${ }^{124,125}$ While there is no debate that potential donors of fecal material should undergo stringent screening, the donor screening protocols have been and, for the foreseeable future, will be subject to change as our experience with FMT therapy increases. International guidelines for donor screening protocols, for example as published by the European FMT working group, may help to reduce the variability of screening protocols used in FMT trials. ${ }^{126}$ In addition to donor screening and selection, other steps in the FMT procedure could be reviewed for optimization. For example, potentially beneficial obligate anaerobic organisms may be lost in the process of FMT preparation unless care is taken to process potential donor material in an anaerobic fashion. Additionally, the best route of administration of the FMT product is unknown, with options including capsules, nasoduodenal infusion and enema.

\section{Microbial metabolites}

Finally, one could bypass the bacterial community and directly supply microbial metabolites, such as the SCFA, acetate, butyrate or propionate. Fecal SCFA concentrations decrease in patients with acute GvHD in proportion to the severity of their disease. ${ }^{46}$ Similarly, the plasma concentrations of butyrate and propionate were reduced in patients who developed chronic GvHD. ${ }^{54}$ Exogenous butyrate supplementation as well as administration of a consortium of 17 known butyrate-producing bacterial strains mitigated murine GvHD in a study by Mathewson and colleagues. ${ }^{66}$ In contrast, a high relative abundance of butyrogenic bacteria in allogeneic HCT with acute GvHD has been associated with steroid-resistance, although luminal butyrate concentrations were not measured.127 These contrasting results highlight that we still have more work to do to understand the biology of butyrate signaling in the post-transplant setting, particularly with respect to GvHD.

\section{Outstanding questions and future perspective}

Considering that the field of microbiome research in hematologic patients is relatively young, significant advances have been made in recent years. Large cohort studies have robustly shown that microbiota injury during allogeneic HCT is common and associated with adverse outcomes. This has already led to some changes in practice - e.g., the favoring of anaerobe-sparing empiric antibiotics in many centers. Results from the first, predominantly single-arm trials have demonstrated potential for microbiota-modulating therapies to improve transplant outcomes (e.g., our recent study of FMT for steroid-refractory acute GvHD), paving the way for eagerly awaited randomized controlled trials that will confirm the clinical efficacy of these therapies in preventing or treating transplantation-related complications. The accessibility of these biological, often already commercially available treatments has facilitated rapid translation from bench to bedside, with a large number of clinical trials currently being undertaken to target dysbiosis in allogeneic HCT recipients. In order to move the field forward in the coming years, several fundamental questions will need to be addressed (summarized inTable 2).
Several microbiome-targeting approaches have shown promising results in preclinical and clinical studies but the key players that underlie this beneficial effect are yet to be defined. Thus far, Blautia as well as butyrate-producing Clostridia appear to be prominent candidates based on fecal microbiome analysis. Still, it is important to keep in mind that there is only partial overlap between the fecal microbiome and the intestinal microbial composition and that the taxa that we have associated with positive transplant outcomes might only be a reflection of a balanced microbiota community. ${ }^{128}$ More invasive sampling (e.g., consecutive colon biopsies) will be needed to confirm the taxa present at the most immunologically active sites within the gastrointestinal tract, and access to these samples will also further enhance our understanding of the working mechanisms of these therapies. Apart from the bacterial constituents of the microbiome, it is going to be important to examine other microbial kingdoms present in the enteric flora such as viruses, including bacteriophages, fungi and archaea. A small study demonstrated that a sterile fecal transplant, filtered from bacterial microbiota, was sufficient to cure Clostridioides difficile infection, implying that non-bacterial microorganisms and/or microbial metabolites are also capable of disease resolution. ${ }^{129}$

In addition to identifying protective bacterial taxa and their related metabolites, the long-term effects of treatment response warrant further investigation. It is not known whether the different therapies discussed here only induce a local and temporary reset of the microbiome or whether they elicit a systemic and durable response to dampen ongoing inflammatory processes. Yoshifuji and colleagues observed that after termination of prebiotics (at day 28 post-HCT), the incidence of lateonset acute GvHD (>day 100) was higher in the prebiotic group than in historical controls, suggesting that more work is needed to understand the potential mechanisms of prebiotic activity after HCT ${ }^{86}$ The same holds true for FMT in which donor bacterial strains are eventually undermined by environmental and/or host factors in modeling the bacterial composition. ${ }^{118}$ This is supported by the finding in our FMT trial that early use of antibiotics after donor FMT appeared to abrogate treatment response. Antibiotic treatment might thus be taken into account in clinical trial design for microbiome-targeting therapies, for example by including an additional/escape intervention in the case of antibiotic treatment. Once intestinal homeostasis is restored, disappearance of donor strains might be less problematic; while taxonomic similarity to the donor's microbiome declined in the study by Moss et al., the functional similarity persisted at a high level. Future studies including long-term follow-up and longitudinal sampling will help to determine the optimal interval for intervention or whether, for example, lifelong dietary restrictions or prebiotic or postbiotic supplementation should be encouraged.

Ideally, in the near future, microbiome analysis should be included in the routine work-up/follow-up for HCT, just as other organ function is measured, since the microbiome can function as a biomarker for transplant-related complications, as well as being potentially modifiable in the pre-transplant period. ${ }^{130,131}$ The microbiome is a significant focus of many research groups internationally, and it is likely that the coming years will continue to yield important results in both the pre-clinical and clinical set- 
Table 2. Outstanding questions and potential investigational approaches.

\section{Key questions $\quad$ Potential investigational approach}

Bacterial composition-focused

Which 'protective' taxa from associative studies are truly beneficial as therapeutics?

Transfer defined bacterial consortia in HCT patients + trials with more invasive sampling especially at time of GrHD diagnosis.

What is the best way to collect additional data to support that change in bacterial composition is indeed the mechanism of clinical improvement?

Add important secondary endpoints to initial studies as well as clinical responses (e.g., fecal engraftment of specific taxa, bacterial diversity, active metabolic pathways, serum and fecal SCFA concentrations).

What is the optimal diet for the patient to produce the optimal microbiome?

Start with observational diet studies, move toward interventional randomized trials.

Metabolite-focused

Which are most important (SCFA, bile acids etc.)?

Where do they need to act for maximum benefit?

Which patient-intrinsic factors influence therapy efficacy (e.g. baseline diversity, presence of specific taxa, level of epithelial damage, genetic factors)?

Mouse studies + trials of 'post-biotic' compounds.

What is the optimal timing and interval for intervention?

Small interventional studies + trials with long-term follow-up.

HCT: hematopoietic cell transplantation; GvHD: graft-versus-host disease; SCFA: short-chain fatty acids.

ting with regard to how we may target the microbiome for maximal benefit for patients.

\section{Disclosures}

$M R M v d B$ has received research support from Seres Therapeutics; has consulted for, received honorarium from or participated in advisory boards for Seres Therapeutics, WindMIL therapeutics, Rheos, Frazier Healthcare Partners, Nektar Therapeutics, Notch Therapeutics, Forty Seven inc., Priothera,
Ceramedix, Lygenesis, Pluto Immunotherapeutics, Magenta Therapeutics, Merck \& Co, Inc., and DKMS Medical Council (Board); has IP Licensing with Seres Therapeutics, Juno Therapeutics, and stock options from Seres and Notch Therapeutics. None of the other authors has anything to disclose.

\section{Contributions}

YvL and KAM wrote the manuscript. MRMvdB and $M D H$ provided critical feedback and edited the manuscript.

\section{References}

1. Jagasia M, Arora M, Flowers ME, et al. Risk factors for acute GVHD and survival after hematopoietic cell transplantation. Blood. 2012;119(1):296-307

2. D'Souza A, Fretham C, Lee SI, et al. Current use of and trends in hematopoietic cell transplantation in the United States. Biol Blood Marrow Transplant. 2020;26(8):e177e182.

3. Gooptu M, Koreth J. Translational and clinical advances in acute graft-versus-host disease. Haematologica. 2020;105(11):25502560.

4. Sonis ST. The pathobiology of mucositis. Nat Rev Cancer. 2004;4(4):277-284.

5. Bennett JE, Dolin R, Blaser MJ, Mandell GL, Douglas RG. Mandell, Douglas, and Bennett's Principles and Practice of Infectious Diseases. 2015. Eighth edition.

6. Zeiser R, Blazar BR. Acute graft-versus-host disease - biologic process, prevention, and therapy. N Engl J Med. 2017;377(22):21672179.

7. Antin JH, Ferrara JL. Cytokine dysregulation and acute graft-versus-host disease. Blood. 1992;80(12):2964-2968.

8. MacMillan ML, Robin M, Harris AC, et al. A refined risk score for acute graft-versus-host disease that predicts response to initial therapy, survival, and transplant-related mortality. Biol Blood Marrow Transplant. 2015;21 (4):761-767.

9. Jagasia M, Zeiser R, Arbushites M, Delaite P, Gadbaw B, Bubnoff NV. Ruxolitinib for the treatment of patients with steroid-refractory GVHD: an introduction to the REACH trials. Immunotherapy. 2018;10(5):391-402.

10. Rinninella E, Raoul P, Cintoni M, et al. What is the healthy gut microbiota composition? A changing ecosystem across age, environment, diet, and diseases. Microorganisms. 2019;7(1):14.

11. Amarasinghe SL, Su S, Dong X, Zappia L, Ritchie ME, Gouil Q. Opportunities and challenges in long-read sequencing data analysis. Genome Biol. 2020;21(1):30.

12. Turnbaugh PJ, Ley RE, Hamady M, FraserLiggett CM, Knight R, Gordon JI. The human microbiome project. Nature. 2007;449(7164):804-810.

13. Falony G, Joossens M, Vieira-Silva S, et al. Population-level analysis of gut microbiome variation. Science. 2016;352(6285):560-564.

14. Zhernakova A, Kurilshikov A, Bonder MJ, et al. Population-based metagenomics analysis reveals markers for gut microbiome composition and diversity. Science. 2016;352 (6285):565-569.

15. Jackson MA, Verdi S, Maxan ME, et al. Gut microbiota associations with common diseases and prescription medications in a population-based cohort. Nat Commun. 2018;9 (1):2655.

16. Hooper LV, Macpherson AJ. Immune adaptations that maintain homeostasis with the intestinal microbiota. Nat Rev Immunol. 2010;10(3):159-169

17. Kamada N, Seo SU, Chen GY, Nunez G. Role of the gut microbiota in immunity and inflammatory disease. Nat Rev Immunol. 2013;13(5):321-335.
18. Yatsunenko T, Rey FE, Manary MJ, et al. Human gut microbiome viewed across age and geography. Nature. 2012;486(7402):222 227.

19. Miller TL, Wolin MJ. Pathways of acetate, propionate, and butyrate formation by the human fecal microbial flora. Appl Environ Microbiol. 1996;62(5):1589-1592.

20. Ridlon JM, Kang DJ, Hylemon PB. Bile salt biotransformations by human intestinal bacteria. J Lipid Res. 2006;47(2):241-259.

21. Correa-Oliveira R, Fachi JL, Vieira A, Sato FT, Vinolo MA. Regulation of immune cell function by short-chain fatty acids. Clin Transl Immunology. 2016;5(4):e73.

22. Tan J, McKenzie C, Potamitis M, Thorburn AN, Mackay CR, Macia L. The role of shortchain fatty acids in health and disease. Adv Immunol. 2014;121:91-119.

23. Smith T. A modification of the method for determining the production of indol by bacteria. J Exp Med. 1897;2(5):543-547.

24. Ridlon JM, Harris SC, Bhowmik S, Kang DJ, Hylemon PB. Consequences of bile salt biotransformations by intestinal bacteria. Gut Microbes. 2016;7(1):22-39.

25. Sun M, Wu W, Chen L, et al. Microbiotaderived short-chain fatty acids promote Th1 cell IL-10 production to maintain intestinal homeostasis. Nat Commun. 2018;9(1):3555

26. Dudakov JA, Hanash AM, van den Brink MR. Interleukin-22: immunobiology and pathology. Annu Rev Immunol. 2015;33: 747-785.

27. Taur Y, Xavier JB, Lipuma L, et al. Intestina domination and the risk of bacteremia in patients undergoing allogeneic hematopoi- 
The post-HCT microbiome: effects and targeted therapy

tic stem cell transplantation. Chin Infect Dis. 2012;55(7):905-914.

28. Ubeda C, Thur Y, Jenq RR, et al. Vancomycin-resistant Enterococcus domenation of intestinal microbiota is enabled by antibiotic treatment in mice and precedes bloodstream invasion in humans. J Chin Invest. 2010;120(12):4332-4341.

29. Thur Y, Coyle K, Schluter J, et al. Reconstitution of the gut microbiota of antibiotic-treated patients by autologous fecal microbiota transplant. Sci Transl Med. 2018;10(460):eaa9489.

30. Peed JU, Comes ALC, Devin SM, et al. Microbiota as predictor of mortality in allogeneric hematopoietic-cell transplantation. $\mathrm{N}$ Engl J Med. 2020;382(9):822-834.

31. Weber D, Jenq RR, Puled JU, et al. Microbiota disruption induced by early use of broad-spectrum antibiotics is an indpendent risk factor of outcome after ilogenic stem cell transplantation. Biol Blood Marrow Transplant. 2017;23(5):845-852.

32. Shone Y, Docampo MD, Pelee JU, et al. Increased GVHD-related mortality with broad-spectrum antibiotic use after allogeneric hematopoietic stem cell transplantaion in human patients and mice. Sci Transl Med. 2016;8(339):339ra371.

33. Simms-Waldrip TR, Sunkersett G, Coughlin LA, et al. Antibiotic-induced depletion of anti-inflammatory clostridia is associated with the development of graft-versus-host disease in pediatric stem cell transplantation patients. Biol Blood Marrow Transplant. 2017;23(5):820-829.

34. Lee SE, Lin JY, Ry DB, et al. Alteration of the intestinal microbiota by broad-spectrum antibiotic use correlates with the occurrence of intestinal graft-versus-host disease. Biol Blood Marrow Transplant. 2019;25(10): 1933-1943.

35. van Vliet MJ, Missing WJ, Dun CA, et al. Chemotherapy treatment in pediatric patients with acute myeloid leukemia receiving antimicrobial prophylaxis leads to a relative increase of colonization with potentially pathogenic bacteria in the gut. Chin Infect Dis. 2009;49(2):262-270.

36. Zwielehner J, Lass l C, Hipper B, et al. Changes in human fecal microbiota due to chemotherapy analyzed by TaqMan-PCR, 454 sequencing and PCR-DGGE fingerprinting. PLo One. 2011;6(12):e28654.

37. Bragi E, Zama D, Nastasi C, et al. Gut microbiota trajectory in pediatric patients undergoing hematopoietic SCT. Bone Marrow Transplant. 2015;50(7):992-998.

38. Galloway-Pena JR, Shi Y, Peterson CB, et al. Gut microbiome signatures are predictive of infectious risk following induction therapy for acute myeloid leukemia. Chin Infect Dis. 2020;71(1):63-71.

39. Montassier E, Gastinne T, Vangay P, et al. Chemotherapy-driven dysbiosis in the intestinal microbiome. Aliment Pharmacol Then. 2015;42(5):515-528.

40. Tour Y, Jenq RR, Perales MA, et al. The effects of intestinal tract bacterial diversity on mortality following allogeneic hematopoietic stem cell transplantation. Blood. 2014;124(7):1174-1182.

41. Jena RR, Thur Y, Devin SM, et al. Intestinal blautia is associated with reduced death from graft-versus-host disease. Biol Blood Marrow Transplant. 2015;21(8):1373-1383.

42. Stein-Thoeringer CK, Nichols KB, Lazrak A, et al. Lactose drives Enterococcus expansion to promote graft-versus-host disease. Science. 2019;366(6469):1143-1149.

43. Holler E, Butzhammer P, Schmid K, et al.
Metagenomic analysis of the stool microbicme in patients receiving allogeneic stem cell transplantation: loss of diversity is associated with use of systemic antibiotics and more pronounced in gastrointestinal graft-versushost disease. Biol Blood Marrow Transplant. 2014;20(5):640-645

44. Bragi E, Zama D, Rampelli S, et al. Early gut microbiota signature of a GvHD in children given allogeneic hematopoietic cell transplantation for hematological disorders. BMC Med Genomics. 2019;12(1):49.

45. lett EE, Jorgensen M, Noguera-Julian M, et al. Associations of the gut microbiome and clinical factors with acute GVHD in allogeneric HSCT recipients. Blood Adv. 2020;4(22):5797-5809.

46. Payer M, Nicolis I, Robin M, et al. Functional and phylogenetic alterations in gut microbiome are linked to graft-versushost disease severity. Blood Adv. 2020;4(9):1824-1832.

47. Jenq RR, Ubeda C, Thur Y, et al. Regulation of intestinal inflammation by microbiota following allogeneic bone marrow transplantaion. J Exp Med. 2012;209(5):902-910.

48. Varelias A, Ormerod KL, Bunting MD, et al. Acute graft-versus-host disease is regulated by an IL-17-sensitive microbiome. Blood. 2017;129(15):2172-2185.

49. Koyama M, Mukhopadhyay P, Schuster IS, et al. MHC class II antigen presentation by the intestinal epithelium initiates graft-versus-host disease and is influenced by the microbiota. Immunity. 2019;51(5):885-898.

50. Eriguchi Y, Takashima S, Oka H, et al. Graftversus-host disease disrupts intestinal microbial ecology by inhibiting Paneth cell production of alpha-defensins. Blood. 2012;120(1):223-231.

51. Paled JU, Devin SM, Staffas A, et al. Intestinal microbiota and relapse after hematopoietic-cell transplantation. J Chin Oncol. 2017;35(15):1650-1659.

52. Harris B, Morjaria SM, Littmann ER, et al. Gut microbiota predict pulmonary infiltrates after allogeneic hematopoietic cell transplantation. Am J Respir Crit Care Med. 2016;194(4):450-463.

53. Hawk BW, Littmann ER, Chaubard JL, et al. Impact of gut colonization with butyrateproducing microbiota on respiratory viral infection following allo-HCT. Blood. 2018;131(26):2978-2986.

54. Markey KA, Schluter J, Comes ALC, et al. The microbe-derived short-chain fatty acids butyrate and propionate are associated with protection from chronic GVHD. Blood. 2020;136(1):130-136.

55. Tamburini FB, Andermann TM, Tkachenko E, Senchyna F, Banaei N, Brat AS. Precision identification of diverse bloodstream pathogens in the gut microbiome. Nat Med. 2018;24(12):1809-1814.

56. Steck N, Hoffman M, Sava IG, et al. Enterococcus faecalis metalloprotease compromises epithelial barrier and contributes to intestinal inflammation. Gastroenterology. 2011;141(3):959-971.

57. Molina MA, Dian AM, Hesse C, et al. Immunostimulatory effects triggered by Enterococcus faecalis CECT7121 probiotic strain involve activation of dendritic cells and interferon-gamma production. CLoS One. 2015;10(5):e0127262.

58. Ubeda C, Bucci V, Caballero S, et al. Intestinal microbiota containing Barnesiella species cures vancomycin-resistant Enterococcus faecium colonization. Infect Immun. 2013;81(3):965-973.

59. Le Roy T, Debedat J, Marque F, et al.

Comparative evaluation of microbiota engraftment following fecal microbiota transfer in mice models: age, kinetic and microbial status matter. Front Microbial. 2018;9:3289.

60. Smillie CS, Sauk J, Givers D, et al. Strain tracking reveals the determinants of bacterial engraftment in the human gut following fecal microbiota transplantation. Cell Host Microbe. 2018;23(2):229-240.

61. Hall AB, Tolonen AC, Xavier RJ. Human genetic variation and the gut microbiome in disease. Nat Rev Genet. 2017;18(11):690699.

62. Abr MC, Buffie CG, Susac B, et al. TLR-7 activation enhances IL-22-mediated colonization resistance against vancomycinresistant enterococcus. Sci Transl Med. 2016;8(327):327ra325.

63. Derrien M, Van Baarlen P, Hooiveld G, Norin E, Muller M, de Voc WM. Modulation of mucosa immune response, tolerance, and proliferation in mice colonized by the mucin-degrader Akkermansia muciniphila. Front Microbial. 2011;2:166

64. Earle KA, Billings G, Signal $M$, et al. Quantitative imaging of gut microbiota spatrial organization. Cell Host Microbe. 2015;18(4):478-488.

65. Ara T, Hashimoto D, Hayase E, et al. Intestinal goblet cells protect against GVHD after allogeneic stem cell transplantation via Lypd8. Sci Transl Med. 2020;12(550): eaaw0720

66. Mathewson ND, Jenq R, Mathew AV, et al. Gut microbiome-derived metabolites modulate intestinal epithelial cell damage and mitingate graft-versus-host disease. Nat Immunol. 2016;17(5):505-513.

67. Swim A, Giver CR, DeFilipp Z, et al. Indole derived from intestinal microbiota act via type I interferon signaling to limit graft-versus-host disease. Blood. 2018;132 (23):2506-2519.

68. Fujiwara $\mathrm{H}$, Docampo $\mathrm{MD}$, Rives $M$, et al. Microbial metabolite sensor GPR43 controls severity of experimental GVHD. Nat Common. 2018;9(1):3674.

69. Kaiko GE, Ry SH, Moues OI, et al. The Colonic Crypt Protects Stem Cells from Microbiota-Derived Metabolites. Cell. 2016;165(7):1708-1720.

70. Hanash AM, Dudakov JA, Ha G, et al. Interleukin-22 protects intestinal stem cells from immune-mediated tissue damage and regulates sensitivity to graft versus host disease. Immunity. 2012;37(2):339-350.

71. Couturier M, Lamarthee B, Arbez J, et al. IL22 deficiency in donor $\mathrm{T}$ cells attenuates murine acute graft-versus-host disease nortality while sparing the graft-versusleukemia effect. Leukemia. 2013;27(7):15271537.

72. Jor E, Yamane S, Hamasaki A, et al. Enteral supplement enriched with glutamine, fiber, and oligosaccharide attenuates experimental colitis in mice. Nutrition. 2013;29(3):549555.

73. Burrello C, Garavaglia F, Cribiu FM, et al. Therapeutic faecal microbiota transplantaion controls intestinal inflammation through IL 10 secretion by immune cells. Nat Common. 2018;9(1):5184.

74. Atarashi K, Tanoue T, Oshima K, et al. Trey induction by a rationally selected mixture of Clostridia strains from the human microbicta. Nature. 2013;500(7461):232-236.

75. Campbell C, McKenney PT, Konstantinovsky D, et al. Bacterial metaboism of bile acids promotes generation of peripheral regulatory $\mathrm{T}$ cells. Nature. 
2020;581(7809):475-479

76. Hang S, Paik D, Yao L, et al. Bile acid metabolites control TH17 and Treg cell differentiation. Nature. 2019;576(7785):143148

77. Reikvam H, Gronningsaeter IS, Mosevoll KA, Lindas R, Hatfield K, Bruserud $\mathrm{O}$. Patients with treatment-requiring chronic graft versus host disease after allogeneic stem cell transplantation have altered metabolic profiles due to the disease and immunosuppressive therapy: potential implication for biomarkers. Front Immunol. 2017:8:1979.

78. Michonneau D, Latis E, Curis E, et al. Metabolomics analysis of human acute graft-versus-host disease reveals changes in host and microbiota-derived metabolites. Nat Commun. 2019;10(1):5695

79. Joshi NM, Hassan S, Jasani P, et al. Bile acid malabsorption in patients with graft-versushost disease of the gastrointestinal tract. Br J Haematol. 2012;157(3):403-407.

80. Ruutu T, Juvonen E, Remberger $M$, et al. Improved survival with ursodeoxycholic acid prophylaxis in allogeneic stem cell transplantation: long-term follow-up of a randomized study. Biol Blood Marrow Transplant. 2014;20(1):135-138

81. Webb BJ, Brunner A, Lewis J, Ford CD, Lopansri BK. Repurposing an old drug for a new epidemic: ursodeoxycholic acid to prevent recurrent Clostridioides difficile infection. Clin Infect Dis. 2019;68(3):498-500.

82. Weber D, Oefner PJ, Dettmer K, et al. Rifaximin preserves intestinal microbiota balance in patients undergoing allogeneic stem cell transplantation. Bone Marrow Transplant. 2016;51(8):1087-1092.

83. Rearigh L, Stohs E, Freifeld A, Zimmer A. De-escalation of empiric broad spectrum antibiotics in hematopoietic stem cell transplant recipients with febrile neutropenia. Ann Hematol. 2020;99(8):1917-1924.

84. Kaleko M, Bristol JA, Hubert S, et al. Development of SYN-004, an oral beta-lactamase treatment to protect the gut microbiome from antibiotic-mediated damage and prevent Clostridium difficile infection. Anaerobe. 2016;41:58-67.

85. de Gunzburg J, Ghozlane A, Ducher A, et al. Protection of the human gut microbiome from antibiotics. J Infect Dis. 2018;217(4): 628-636.

86. Yoshifuji K, Inamoto $\mathrm{K}$, Kiridoshi $\mathrm{Y}$, et al. Prebiotics protect against acute graft-versushost disease and preserve the gut microbiota in stem cell transplantation. Blood Adv. 2020;4(19):4607-4617.

87. Iyama S, Sato T, Tatsumi H, et al. Efficacy of enteral supplementation enriched with glutamine, fiber, and oligosaccharide on mucosal injury following hematopoietic stem cell transplantation. Case Rep Oncol. 2014;7(3): 692-699.

88. Ladas EJ, Bhatia M, Chen L, et al. The safety and feasibility of probiotics in children and adolescents undergoing hematopoietic cell transplantation. Bone Marrow Transplant. 2016;51(2):262-266.

89. Sadanand A, Newland JG, Bednarski JJ. Safety of probiotics among high-risk pediatric hematopoietic stem cell transplant recipients. Infect Dis Ther. 2019;8(2):301306

90. Gerbitz A, Schultz M, Wilke A, et al. Probiotic effects on experimental graft-versus-host disease: let them eat yogurt. Blood. 2004:103(11):4365-4367.

91. Le Bastard Q, Ward T, Sidiropoulos D, et al. Fecal microbiota transplantation reverses antibiotic and chemotherapy-induced gut dysbiosis in mice. Sci Rep. 2018;8(1):6219.

92. Battipaglia G, Malard F, Rubio MT, et al. Fecal microbiota transplantation before or after allogeneic hematopoietic transplantation in patients with hematologic malignancies carrying multidrug-resistance bacteria. Haematologica. 2019;104(8):1682-1688.

93. Innes AJ, Mullish BH, Fernando F, et al. Faecal microbiota transplant: a novel biological approach to extensively drug-resistant organism-related non-relapse mortality. Bone Marrow Transplant. 2017;52(10):1452-1454.

94. Kolodziejczyk AA, Zheng D, Elinav E. Dietmicrobiota interactions and personalized nutrition. Nat Rev Microbiol. 2019;17(12): $742-753$

95. Li X, Lin Y, Li X, et al. Tyrosine supplement ameliorates murine aGVHD by modulation of gut microbiome and metabolome. EBioMedicine. 2020;61:103048,

96. D'Amico F, Biagi E, Rampelli S, et al. Enteral nutrition in pediatric patients undergoing hematopoietic SCT promotes the recovery of gut microbiome homeostasis. Nutrients. 2019;11(12):2958

97. Andersen S, Staudacher H, Weber N, et al Pilot study investigating the effect of enteral and parenteral nutrition on the gastrointestinal microbiome post-allogeneic transplantation. Br J Haematol. 2020;188(4):570-581.

98. Gonzales F, Bruno B, Alarcon Fuentes M, et al. Better early outcome with enteral rather than parenteral nutrition in children undergoing MAC allo-SCT. Clin Nutr. 2018;37(6 Pt A):2113-2121.

99. Beckerson J, Szydlo RM, Hickson M, et al Impact of route and adequacy of nutritional intake on outcomes of allogeneic haematopoietic cell transplantation for haematologic malignancies. Clin Nutr. 2019;38(2):738-744

100. Segal L, Opie RS. A nutrition strategy to reduce the burden of diet related disease: access to dietician services must complement population health approaches. Front Pharmacol. 2015;6:160.

101. Johnson AJ, Vangay P, Al-Ghalith GA, et al. Daily sampling reveals personalized dietmicrobiome associations in humans. Cell Host Microbe. 2019;25(6):789-802.

102. McFarland LV. Use of probiotics to correct dysbiosis of normal microbiota following disease or disruptive events: a systematic review. BMJ Open. 2014:4(8):e005047.

103. Suez J, Zmora N, Zilberman-Schapira G, et al. Post-antibiotic gut mucosal microbiome reconstitution Is impaired by probiotics and improved by autologous FMT. Cell. 2018;174(6):1406-1423.

104. Cohen SA, Woodfield MC, Boyle N, Stednick Z, Boeckh M, Pergam SA. Incidence and outcomes of bloodstream infections among hematopoietic cell transplant recipients from species commonly reported to be in over-the-counter probiotic formulations. Transpl Infect Dis. 2016;18 (5):699-705

105. Ambesh P, Stroud S, Franzova E, et al. Recurrent Lactobacillus bacteremia in a patient with leukemia. J Investig Med High Impact Case Rep. 2017;5(4) 2324709617744233

106. Mehta A, Rangarajan S, Borate U. A cautionary tale for probiotic use in hematopoietic SCT patients-Lactobacillus acidophilus sepsis in a patient with mantle cell lymphoma undergoing hematopoietic SCT. Bone Marrow Transplant. 2013;48(3):461-462.

107. Salminen MK, Rautelin H, Tynkkynen S, et al. Lactobacillus bacteremia, clinical signifi- cance, and patient outcome, with special focus on probiotic L. rhamnosus GG. Clin Infect Dis. 2004;38(1):62-69.

108. Vahabnezhad E, Mochon AB, Wozniak LJ, Ziring DA. Lactobacillus bacteremia associated with probiotic use in a pediatric patient with ulcerative colitis. J Clin Gastroenterol. 2013;47(5):437-439

109. Robin F, Paillard C, Marchandin H, Demeocq F, Bonnet R, Hennequin C. Lactobacillus rhamnosus meningitis following recurrent episodes of bacteremia in a child undergoing allogeneic hematopoietic stem cell transplantation. J Clin Microbiol. 2010;48(11):4317-4319.

110. Gorshein E, Wei C, Ambrosy S, et al Lactobacillus rhamnosus GG probiotic enteric regimen does not appreciably alter the gut microbiome or provide protection against GVHD after allogeneic hematopoietic stem cell transplantation. Clin Transplant. 2017;31(5).

111. DeFilipp Z, Peled JU, Li S, et al. Third-party fecal microbiota transplantation following allo-HCT reconstitutes microbiome diversity. Blood Adv. 2018;2(7):745-753.

112. Bilinski J, Grzesiowski P, Sorensen N, et al. Fecal microbiota transplantation in patients with blood disorders inhibits gut colonization with antibiotic-resistant bacteria: results of a prospective, single-center study. Clin Infect Dis. 2017;65(3):364-370.

113. Neemann K, Eichele DD, Smith PW, Bociek R, Akhtari M, Freifeld A. Fecal microbiota transplantation for fulminant Clostridium difficile infection in an allogeneic stem cell transplant patient. Transpl Infect Dis. 2012;14(6):E161-165.

114. de Castro CG Jr, Ganc AJ, Ganc RL, Petrolli MS, Hamerschlack N. Fecal microbiota transplant after hematopoietic SCT: report of a successful case. Bone Marrow Transplant. 2015;50(1):145.

115. Mittal C, Miller N, Meighani A, Hart BR John A, Ramesh M. Fecal microbiota transplant for recurrent Clostridium difficile infection after peripheral autologous stem cell transplant for diffuse large B-cell lymphoma. Bone Marrow Transplant. 2015;50(7):1010.

116. Webb BJ, Brunner A, Ford CD, Gazdik MA Petersen FB, Hoda D. Fecal microbiota transplantation for recurrent Clostridium difficile infection in hematopoietic stem cell transplant recipients. Transpl Infect Dis. 2016;18(4):628-633.

117. Bluestone H, Kronman MP, Suskind DL. Fecal microbiota transplantation for recurrent Clostridium difficile infections in pediatric hematopoietic stem cell transplant recipients. J Pediatric Infect Dis Soc. 2018;7(1):e6-e8.

118. Moss EL, Falconer SB, Tkachenko E, et al Long-term taxonomic and functional divergence from donor bacterial strains following fecal microbiota transplantation in immunocompromised patients. PLoS One. 2017;12 (8): $\mathrm{e} 0182585$

119. Kakihana K, Fujioka Y, Suda W, et al. Fecal microbiota transplantation for patients with steroid-resistant acute graft-versus-host disease of the gut. Blood. 2016;128(16):2083 2088

120. Spindelboeck W, Schulz E, Uhl B, et al. Repeated fecal microbiota transplantations attenuate diarrhea and lead to sustained changes in the fecal microbiota in acute, refractory gastrointestinal graft-versus-hostdisease. Haematologica. 2017;102(5):e210e213.

121. Oi X, Li X, Zhao Y, et al. Treating steroid 
refractory intestinal acute graft-vs.-host disease with fecal microbiota transplantation: a pilot study. Front Immunol. 2018;9:2195

122. Kaito S, Toya T, Yoshifuji K, et al. Fecal microbiota transplantation with frozen capsules for a patient with refractory acute gut graft-versus-host disease. Blood Adv. 2018;2(22):3097-3101.

123. van Lier YF, Davids M, Haverkate NJE, et al. Donor fecal microbiota transplantation ameliorates intestinal graft-versus-host disease in allogeneic hematopoietic cell transplant recipients. Sci Transl Med. 2020;12(556): eaaz8926.

124. DeFilipp Z, Bloom PP, Torres Soto M, et al. Drug-Resistant E. coli bacteremia transmitted by fecal microbiota transplant. $\mathrm{N}$ Engl J Med. 2019;381(21):2043-2050.
125. Bilinski J, Lis K, Tomaszewska A, et al. Eosinophilic gastroenteritis and graft-versushost disease induced by transmission of Norovirus with fecal microbiota transplant. Transpl Infect Dis. 2021;23(1):e13386.

126. Cammarota G, Ianiro G, Tilg H, et al European consensus conference on faecal microbiota transplantation in clinical practice. Gut. 2017;66(4):569-580

127. Golob JL, DeMeules MM, Loeffelholz T, et al. Butyrogenic bacteria after acute graft-versus-host disease (GVHD) are associated with the development of steroid-refractory GVHD. Blood Adv. 2019;3(19):2866-2869.

128.Zmora N, Zilberman-Schapira G, Suez J, et al. Personalized gut mucosal colonization resistance to empiric probiotics is associated with unique host and microbiome features.
Cell. 2018;174(6):1388-1405.e21.

129. Ott SJ, Waetzig GH, Rehman A, et al. Efficacy of sterile fecal filtrate transfer for treating patients with Clostridium difficile infection. Gastroenterology. 2017;152(4): 799-811.

130. Han L, Zhang H, Chen S, et al. Intestinal microbiota can predict acute graft-versushost disease following allogeneic hematopoietic stem cell transplantation. Biol Blood Marrow Transplant. 2019;25(10): 1944-1955.

131. Kusakabe S, Fukushima K, Maeda T, et al. Pre- and post-serial metagenomic analysis of gut microbiota as a prognostic factor in patients undergoing haematopoietic stem cell transplantation. Br J Haematol. 2020;188 (3):438-449. 